\title{
Primary leiomyosarcoma of the lung presenting with a persistent pneumothorax
}

\author{
SIMON CAPEWELL, JOHN N WEBB, GRAHAM K CROMPTON \\ From the Northern General Hospital, Edinburgh
}

Primary leiomyosarcoma of the lung is a malignant tumour arising from smooth muscle. It is rare and only two cases were reported in one recent series of 6000 lung tumours. ${ }^{1}$ It is one of the least common types of lung sarcoma. ${ }^{2}$ Most primary leiomyosarcomas originate in the hilar region in relation to the main bronchi or pulmonary vessels and only a few originate more peripherally. ${ }^{3}$ This rare tumour can mimic bronchial carcinoma and present with local or systemic symptoms, or it may be discovered as an incidental finding on a routine chest radiograph. ${ }^{1}$ Symptoms may include shortness of breath, chest pain, sputum, haemoptysis, and weight loss. ${ }^{45}$ Clubbing is rarely present and pneumothorax as the presenting feature of a leiomyosarcoma has not, to our knowledge, been reported previously.

\section{Case report}

A 72 year old retired transport manager was referred to hospital with a two week history of shortness of breath and dull, intermittent left sided chest pain. He had smoked 20 cigarettes a day and had had pneumonia 20 years previously. He was a well nourished man, with appreciable finger clubbing but no lymphadenopathy. The breath sounds were diminished on auscultation of the left side of the thorax, and chest radiography confirmed a shallow left pneumothorax with no visible abnormalities in the pleura, lungs, or mediastinum (fig 1). Haemoglobin concentration, red cell indices, and white cell count were all normal, as were plasma urea and electrolyte concentrations and the results of tests of liver function. The $\mathrm{FEV}_{1}$ was $2 \cdot 3$ litres and the vital capacity (VC) was $3 \cdot 81$.

Despite the consecutive insertion of three intercostal catheters with underwater seal drainage, the pneumothorax persisted for three weeks. Kaolin pleurodesis was therefore performed under general anaesthesia. The intercostal drain was removed after eight days, at which time the chest radiograph showed a small persistent pneumothorax at the apex and at the base. Eight weeks later readmission to hospital was necessary because of increasing shortness of breath, left sided chest tightness, haemoptysis, and weight loss. A chest radiograph showed a left basal effusion with a fluid level and a second loculated effusion and an air space in the upper zone. Almost $700 \mathrm{ml}$ of blood stained fluid was removed from the basal effusion. The protein content was $41 \mathrm{~g} / 1$ and cytological examination was reported to show polymorphs, lymphocytes, macrophages, and reactive serosal cells, but no

Address for reprint requests: Dr S Capewell, Department of Medicine, Royal Infirmary, Edinburgh EH. malignant cells. Five sputum samples were examined for malignant cells, but none were seen.

Four months after initial presentation the patient had become much more short of breath and had continued to lose weight. Cytological examination of sputum at this stage showed clusters of malignant cells "of an unusual type." Cytological examination of the pleural fluid was once more unhelpful. Symptoms were relieved with opiate drugs and death occurred five months after the initial presentation with pneumothorax.

At necropsy there was partial collapse of the left lung with loculated pleural effusions containing haemorrhagic fluid. In the left lower lobe diffuse whitish tumour tissue extended from the hilum to the diaphragmatic and mediastinal aspects of the visceral pleura but it had not invaded a bronchus or major pulmonary vessel. The tumour also extended medially and superiorly to form nodular masses on the outer aspect of the parietal pericardium and surrounding the arch of the aorta, being applied to the medial aspect of the lung at this

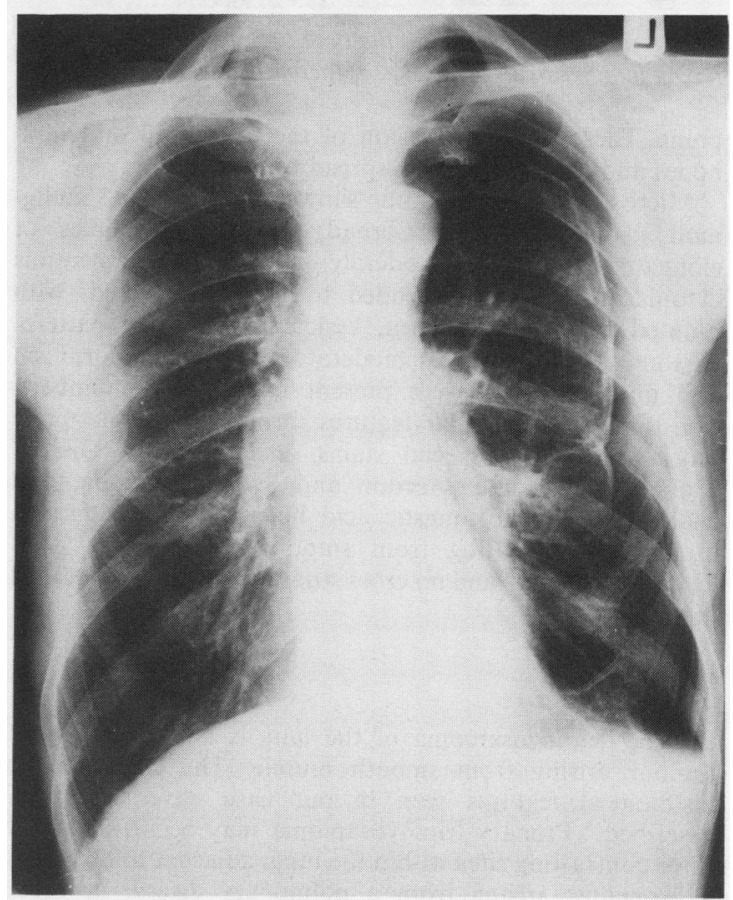

Fig 1 Chest radiograph at presentation showing a shallow left sided pneumothorax. 


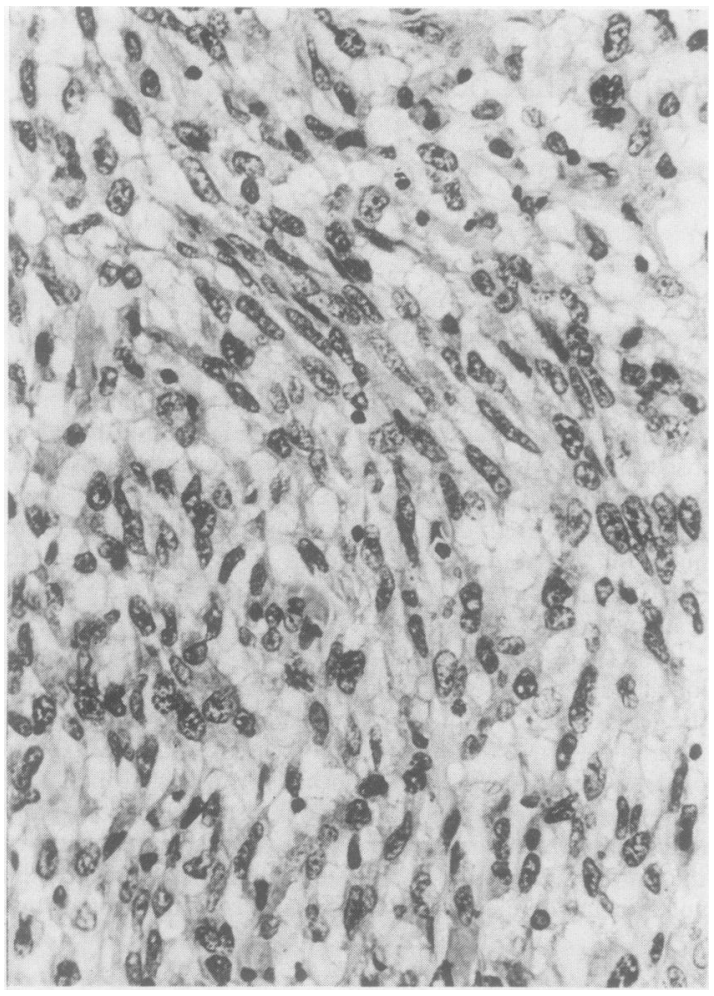

Fig 2 Photomicrograph of the lung tumour showing interlacing bundles of spindle cells. (Haematoxylin and eosin.)

point. There was no invasion of the chest wall or lymph nodes and no extrathoracic spread of the tumour.

Microscopy of the tumour showed a spindle cell malignant neoplasm forming broad, interlacing bundles of elongated cells with moderately abundant filamentous cytoplasm. The nuclei tended to be cigar shaped, with blunted ends and an open, vesicular chromatin pattern. In some areas there was moderate nuclear pleomorphism and mitotic figures were present in moderate numbers. The tumour had similar features throughout in the many blocks examined. Special stains, including van Gieson, Masson's trichrome, Gordon and Sweet's reticulin, and Mallory's phospho-tungstic acid haematoxylin, suggested a tumour originating from smooth muscle-that is, a leiomyosarcoma - and no cross striations could be identified (fig 2).

\section{Discussion}

Primary leiomyosarcoma of the lung is a rare malignant tumour arising from smooth muscle. The characteristic histological features seen in our case have been well described. ${ }^{3}$ Primary leiomyosarcoma may occur in one of three contrasting sites within the lung: adjacent to or within a bronchus, arising from a pulmonary artery, or more peripherally in the lung parenchyma as an "intrapulmonary" leiomyosarcoma. ${ }^{36}$

Intrapulmonary primary leiomyosarcoma related to bronchi may present with dyspnoea, chest pain, cough, sputum, haemoptysis, or weight loss. ${ }^{45}$ Occasionally a pulmonary opacity may be discovered as an incidental finding on a chest $\vec{F}$ radiograph. ${ }^{1}$ The finger clubbing observed in our patient is $\stackrel{0}{\rightarrow}$ very unusual. ${ }^{5}$ Primary leiomyosarcoma presenting with persistent pneumothorax has not, to our knowledge, been $\bar{\sigma}$ described previously. The most frequent radiological finding $\frac{\bar{\omega}}{\sigma}$ with intrapulmonary primary leiomyosarcoma is a rounded, $\mathbb{D}$ well circumscribed mass within the lung or adjacent to the hilum. ${ }^{1}$ Bronchial and endobronchial primary leiomyo- $\infty$ sarcoma may, of course, produce lobar or segmental col- $\overrightarrow{0}$ lapse. This rare tumour may mimic bronchial carcinoma both clinically and radiologically. ${ }^{15}$ In contrast, primary $\vec{\omega}$ leiomyosarcoma of the pulmonary artery usually presents as a loculated hilar mass on the chest radiograph associated $\underset{\vec{x}}{\vec{x}}$ with progressive right sided heart failure ${ }^{68}$ and may even mimic acute pulmonary embolism. ${ }^{9}$ Pneumothorax has been $\overrightarrow{\vec{\phi}}$ described as a complication of primary uterine leiomyosarcoma with lung metastases, ${ }^{10}$ but not of primary $\stackrel{+}{c}$ leiomyosarcoma of the lung.

The diagnosis of primary leiomyosarcoma of the lung usually requires thoracotomy ${ }^{11}$ but can occasionally be $\vec{\longrightarrow}$ made at bronchoscopy. ${ }^{5712}$ Surgical resection offers the only curative treatment, radiotherapy and chemotherapy being ineffective. ${ }^{111}$ The tumour is locally invasive but $\stackrel{\text { क }}{\rightarrow}$ remains confined to the lung for much longer than bronchial $\vec{\bullet}$ carcinoma. ${ }^{3}$ Metastases occur late and in less than one third $\infty$ of cases. ${ }^{45}$ The prognosis is worse with larger tumours, extensive local spread, or a high mitotic rate. ${ }^{2}$

The first successful surgical resection of primary leiomyosarcoma of the lung was performed in $1936^{7}$ and several long term survivors have been described, with a five year survival rate approaching $50 \%$ after resection. ${ }^{1511}$

We are most grateful to Miss $\mathbf{J}$ Holywell for typing the manuscipt.

\section{References}

1 Cameron EJW. Primary sarcoma of the lung. Thorax 1975;30:516-20.

2 Gebauer $C$. The post-operative prognosis of primary pulmonary sarcoma. A review with a comparison between the histological forms and the other primary endothoracic sarcomas based on 474 cases. Scand J Thorac Cardiovasc Surg 1982;16:91-9.

3 Spencer H. Pathology of the lung. 4th ed. London: Pergamon $ᄋ$ Press, 1985:983.

4 Agnos, JW, Starkey GWB. Primary leiomyosarcoma and $\frac{D}{O}$ leiomyoma of the lung. $N$ Engl $J$ Med 1958;258:12-7. 5 Ramanathan T. Primary leiomyosarcoma of the lung. Thorax $\mathrm{N}$

6 Pain JA, Sayer RE. Primary leiomyosarcoma of the pulmonary $N$ artery. Eur J Respir Dis 1984;65:139-43.

7 Morgan PGM, Ball J. Pulmonary leiomyosarcomas. Br J Dis Chest 1980;74:245-52.

8 Moffat RE, Chang $\mathrm{CH}$, Slaven JE. Roentgen considerations in primary pulmonary artery sarcoma. Radiology 1972;104:283-8.

9 Olsson HE, Spitzer RM, Erston WF. Primary and secondary pulmonary artery neoplasia mimicking acute pulmonary ${ }^{+}$ embolism. Radiology 1976;118:49-54.

10 Mehzad M. Leiomyosarcoma of the uterus presenting with pneumothorax. Br J Dis Chest 1977;71:132-4.

11 Yellin A, Rosenman Y, Leiberman Y. Review of smooth muscle $\frac{\rho}{\Phi}$ tumours of the lower respiratory tract. Br J Dis Chest 1984;78:337-51.

12 Sawada K, Fukuma S, Seki Y, et al. Cytological features of primary leiomyosarcoma of the lung. Report of a case diagnosed $\delta$ by bronchial brushing procedure. Acta Cytol (Baltimore) 1977;21:770-3. 